\title{
Dynamic Oxygen Motion in Irradiated-Annealed High Temperature Superconducting Wire
}

\author{
Yi Zhang ${ }^{1}$, M. W. Rupich ${ }^{2}$, Vyacheslav Solovyov ${ }^{3}$, Qiang $\mathrm{Li}^{4}$ and Amit Goyal ${ }^{*}$ \\ 1. Research and Education in Energy, Environment \& Water (RENEW), State University of New York \\ (SUNY), Buffalo, NY, USA. \\ 2. AMSC, Massachusetts, USA. \\ 3. Brookhaven Technology Group, Stony Brook, NY, USA. \\ 4. Brookhaven National Laboratory, Upton, NY, USA. \\ * Corresponding author: agoyal@buffalo.edu
}

Research over the past years has shown that proton and ion irradiation can significantly improve pinning in second-generation (2G) HTS wire by producing highly engineered defects [1-3]. However, the enhancement in $J_{c}$ usually comes with a cost of depression of critical temperature $T_{c}$. The degradation can be partially recovered by post-annealing process [4]. The film quality and superconducting properties can be improved by altering the oxygen disorder and nanoscale defects after annealing. Although irradiation defects have been studied over a full range of atomic, electronic structures and physical properties, a detailed atomic level TEM analysis on the annealing effects is still needed.

Here, we use the atomically resolved electron energy loss spectroscopy (EELS) to locate the oxygen vacancies in the planes and chains of YBCO. A $1.2 \mu \mathrm{m}$ thick MOD REBCO-based $2 \mathrm{G}$ wire was irradiated with $18-\mathrm{MeV} \mathrm{Au}^{5+}$ ions to a dose of $2 \times 10^{11} \mathrm{Au} / \mathrm{cm}^{2}$ along the $c$-axis of YBCO film [5]. Prior to the irradiation, the wire sample has a $77 \mathrm{~K}$, self-field $I_{c}$ of around $385 \mathrm{~A} / \mathrm{cm}-\mathrm{w}$. In comparison, the value decreased to $320 \mathrm{~A} / \mathrm{cm}-\mathrm{w}$ after irradiation. The irradiated samples were then annealed in oxygen atmosphere at various temperatures, which led to an enhancement in $77 \mathrm{~K}$, self-field $I_{c}$ to around 358 A/cm-w.

We present a detailed investigation of the oxygen behavior during the irradiation and annealing processes by probing electronic structure of different sites in YBCO matrix. We extracted the $\mathrm{Cu}-L_{2,3}$ edge spectra from different layers of YBCO structure in the non-irradiated, as-irradiated, and annealed samples to clarify the location of oxygen vacancies. The $\mathrm{Cu} L_{3}$ and $L_{2}$ white lines are very sensitive to the change in valence states. The chain layer spectrum in the non-irradiated film (Figure 1a) shows splitting peaks at $934.5 \mathrm{eV}$ (peak $a$ ) and $937.2 \mathrm{eV}$ (peak $b$ ), due to the existence of mixed valence states of $\mathrm{Cu}^{2+}$ (peak $a$ ) and $\mathrm{Cu}^{1+}$ (peak $b$ ). The plane layer shows only peak $a$ which is consistent with the previous reported $\mathrm{YBCO}_{7}$ data [6]. In the as-irradiated film (Figure 1b), the chain layer shows a strong main peak $b$ with a weak shoulder peak $a$, which corresponds to predominant $\mathrm{Cu}^{1+}$ ions in $\mathrm{Cu}-\mathrm{O}$ chains. The $\mathrm{CuO}_{2}$ plane layer shows splitting peaks $a$ and $b$, which indicates the coexistence of $\mathrm{Cu}^{2+}$ and $\mathrm{Cu}^{1+}$ oxidation states. The previous research showed that plane layer $\mathrm{Cu}-L_{2,3}$ does not show a significant change in $\mathrm{YBCO}_{7-\delta}$ from $\delta=1$ (full chains) to $\delta=0$ (empty chains). The splitting plane layer spectrum in the as-irradiated film can be caused by the oxygen displacement in both planes and chains. After annealing in oxygen atmosphere (Figure 1c), the chain layers still show main peak $b$ with a shoulder peak $a$. However, the plane layers have the similar $\mathrm{Cu}$ edges as non-irradiated sample. The results indicate the $\mathrm{O}$ doping during annealing process is preferred to reoccupy the $\mathrm{O}$ vacancy sites in plane layers. 
In conclusion, we performed a detailed analysis of the reversible dynamic behavior of oxygen vacancies in YBCO film during irradiation-annealing processes. The low energy heavy ion irradiation creates oxygen point defects in both chain and plane layer of YBCO film. The plane sites oxygen vacancies were recovered in post-annealing process. The suppressed $T_{c}$ and self-field $I_{c}$ in the irradiated samples were partially recovered after annealing. The variation tread is consistent with the dynamic behavior of oxygen defects, which indicates the strong influence of plane site oxygen vacancies on the superconductivity properties in the irradiated HTS wires [7].

\section{References:}

[1] Van Dover, R. B. et al., Nature 342 (1989), p. 55.

[2] Civale, L. et al., Physical Review Letters 65 (1990), p. 1164.

[3] Sauerzopf, F. M. et al., Physical Review B 43 (1991), p. 3091.

[4] Vlcek, B. M. et al., Physical Review B 48 (1993), p. 4067.

[5] Rupich, M. W. et al., IEEE Transactions on Applied Superconductivity 26 (2016), p. 1.

[6] Gauquelin, N. et al., Nature Communications 5 (2014), p. 4275.

[7] The authors acknowledge funding from the DOE/EERE under contract No: DE-EE0007870, and support from U.S. Department of Energy Office of Energy Efficiency \& Renewable Energy, Advanced Manufacturing Office for this program.

(a)

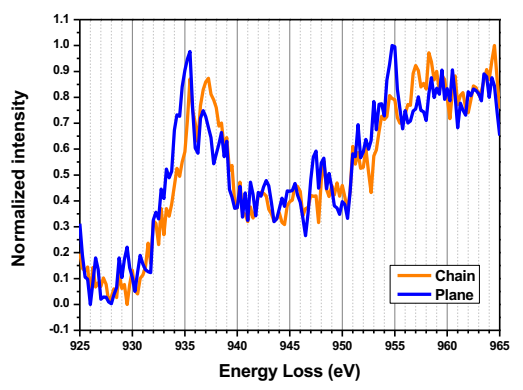

(b)

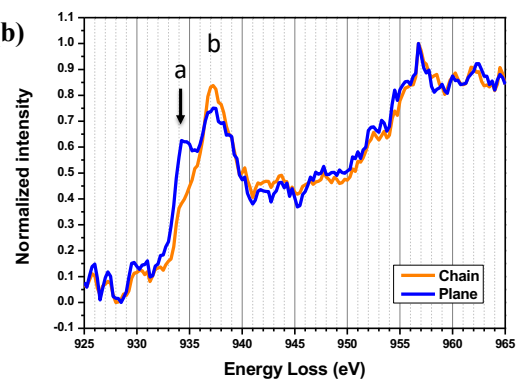

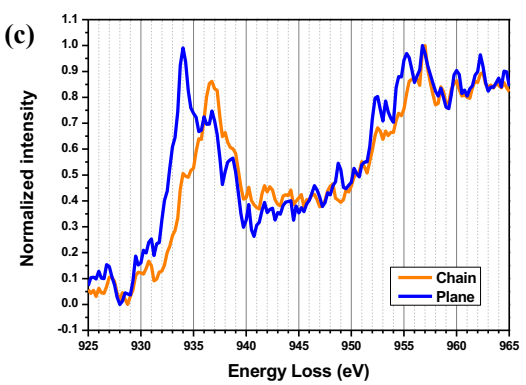

Figure 1. $\mathrm{Cu}-L_{2,3}$ edge spectra are extracted from different layers of YBCO in (a) non-irradiated, (b) asirradiated, and (c) annealed films. In non-irradiated film, the spectra show splitting $\mathrm{Cu}-L_{3}$ edge in chain layer and peak $a$ in plane layer, which is consistent with the $\mathrm{YBCO}_{7}$ structure. In (b), the chain layer shows one peak, which corresponds to $\mathrm{Cu}^{1+}$ ions in $\mathrm{Cu}-\mathrm{O}$ chains. $\mathrm{Cu} L_{3}$ edge of $\mathrm{CuO}_{2}$ plane layer shows splitting peaks $a$ and $b$, indicating the coexistence of $\mathrm{Cu}^{2+}$ and $\mathrm{Cu}^{1+}$ oxidation states. (c) After annealing, the chain layers still show peak $b$, but the plane layers have only one strong peak $a$. 